Research Article

\title{
Possible Probability and Irreducibility of Balanced Nontransitive Dice
}

\author{
Injo Hur $(\mathbb{D}$ and Yeansu Kim \\ Department of Mathematics Education, Chonnam National University, Gwangju City, Republic of Korea \\ Correspondence should be addressed to Yeansu Kim; ykim@jnu.ac.kr
}

Received 13 November 2020; Revised 11 January 2021; Accepted 15 January 2021; Published 29 January 2021

Academic Editor: Antonio Di Crescenzo

Copyright ( 92021 Injo Hur and Yeansu Kim. This is an open access article distributed under the Creative Commons Attribution License, which permits unrestricted use, distribution, and reproduction in any medium, provided the original work is properly cited.

We construct irreducible balanced nontransitive sets of $n$-sided dice for any positive integer $n$. One main tool of the construction is to study so-called fair sets of dice. Furthermore, we also study the distribution of the probabilities of balanced nontransitive sets of dice. For a lower bound, we show that the winning probability can be arbitrarily close to $1 / 2$. We hypothesize that the winning probability cannot be more than $(1 / 2)+(1 / 9)$, and we construct a balanced nontransitive set of dice whose probability is $(1 / 2)+(13-\sqrt{153} / 24) \approx(1 / 2)+(1 / 9.12)$.

\section{Introduction}

A nontransitive triple of dice consists of three dice, labeled $A, B$, and $C$, with the property that all three probabilities that $A$ rolls higher than $B$, that $B$ rolls higher than $C$, and that $C$ rolls higher than $A$ are greater than $1 / 2$. We write this as $P(A>B)>(1 / 2), P(B>C)>(1 / 2)$, and $P(C>A)>(1 / 2)$. It is called nontransitive because if we define the relation $X>Y$ as $P(X>Y)>(1 / 2)$ for $X, Y \in\{A, B, C\}$, then the relation $>$ is not transitive. For example, the following set of 6-sided dice is nontransitive since $P(A>B)=P(B>C)=P(C>A)=(19 / 36)$ :
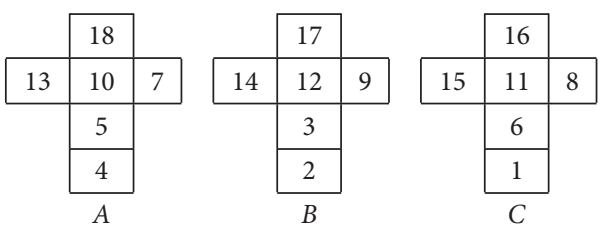

Note that nontransitive sets of dice are first introduced by Gardner [1], further studied in [2, 3], and have been generalized in several directions (see [4-14]). In [14], Schaefer and Schweig constructed balanced nontransitive sets of $n$-sided dice for any positive integer $n \geq 3$ (see below for the definition of "balanced" and other terms). Main idea of the construction in [14] is to combine several balanced nontransitive sets of dice. Therefore, the sets of dice that are constructed in [14] are reducible, and Schaefer and Schweig [14] question whether there exist balanced irreducible nontransitive sets of $n$-sided dice for all $n$. One main purpose of the paper is to construct irreducible nontransitive sets of $n$-sided dice for any positive integer $n$. Our main idea of the construction is to use so-called fair sets of 2-sided dice. Here, being fair means that probabilities $P(A>B), P(B>C)$, and $P(C>A)$ are all $1 / 2$. Although we used 2-sided dice to construct new sets of dice, understanding fair sets of $n$-sided dice for any positive integer $n$ seems to be an important step to understand all irreducible balanced nontransitive dice. We also study fair sets of $n$-sided dice for any positive integer $n$.

The second purpose of the paper is to study possible probabilities of balanced nontransitive sets of $n$-sided dice, i.e., possible value of $P(A>B)$. As far as we know, previous known constructions of balanced nontransitive sets of dice have probability $(1 / 2)<P(A>B)<(3 / 5)=(1 / 2)+(1 / 10)$. We first show that there exist balanced nontransitive sets of dice such that $P(A>B)-(1 / 2)>0$ is arbitrarily small, i.e., $1 / 2$ is a sharp lower bound for balanced nontransitive set of 
dice. We conjecture that, in general, $(1 / 2)<P(A>B)<(1 / 2)+(1 / 9)$. To support the conjecture, we first explicitly calculate and prove that both the probability $P(A>B)$ in our paper and the one in [14] are less than $(1 / 2)+(1 / 9)$. We also provide another new construction of a balanced nontransitive set of dice such that $P(A>B) \approx(1 / 2)+(1 / 9.12)$, which is less than $(1 / 2)+(1 / 9)$, and is, as far as we know, the maximum among known constructions of balanced nontransitive sets of dice.

Let us now describe the content of our paper. After we introduce notation and preliminaries in Section 2, we study fair sets of dice in Section 3. In Section 4, we provide construction of irreducible balanced sets of $n$-sided dice for any positive integer $n$ using fair sets of 2 -sided dice. In Section 5, we calculate and study possible probability $P(A>B)$ for balanced nontransitive sets of dice.

\section{Notation and Preliminaries}

We briefly recall definitions and notations in [14] that we are going to use.

Definition 1. Fix an integer $n>0$. A set of $n$-sided dice is a collection of three pairwise-disjoint sets $A, B$, and $C$ with $|A|=|B|=|C|=n$ and $A \cup B \cup C=\{1, \ldots, 3 n\}$. We think of dice $A, B$, and $C$ as being labeled with the elements of $A, B$, and $C$, respectively, and we assume that each die is fair (i.e., the probability of rolling any one of its numbers is $1 / n$ ).

Definition 2. A set of dice is called as follows:

(i) Balanced if $P(A>B)=P(B>C)=P(C>A)$

(ii) Nontransitive if each of $P(A>B), P(B>C)$, and $P(C>A)$ exceeds $1 / 2$

(iii) Fair if $P(A>B)=P(B>C)=P(C>A)=(1 / 2)$

Definition 3. If $D=(A, B, C)$ is a set of $n$-sided dice, define a word $\sigma(D)$ by the following rule: the $i^{\text {th }}$ letter of $\sigma(D)$ corresponds to the die on which the number $i$ labels a side.

For a word $\sigma$, the number of letters in the word, which we call the length of $\sigma$, is denoted by $|\sigma|$. A number of $A$ (resp. $B$, $C)$ in $\sigma$ is denoted by $|A|_{\sigma}$ (resp. $|B|_{\sigma},|C|_{\sigma}$ ). For example, if $\sigma$ corresponds to a set of $n$-sided dice, $|A|_{\sigma}=|B|_{\sigma}=|C|_{\sigma}=n$.

Let $D=(A, B, C)$ be a set of $n$-sided dice and $\sigma$ be its corresponding word (Definition 3 ). Then, we denote by $P_{D}(A>B)$ or $P_{\sigma}(A>B)$ the probability that the number rolled on $A$ is greater than the number rolled on $B$ when we roll $A$ and $B$. Similarly, we define $P_{D}(B>C), P_{\sigma}(B>C), P_{D}(C>A)$, and $P_{\sigma}(C>A)$.

Definition 4. For a set of $n$-sided dice $D$ (and its corresponding word $\sigma$ with length $3 n)$, we let $N_{D}(A>B):=n^{2} P_{D}(A>B), N_{D}(B>C):=n^{2} P_{D}(B>C)$ and $N_{D}(C>A):=n^{2} P_{D}(C>A)$. Similarly, we also define $N_{\sigma}(A>B), N_{\sigma}(B>C)$, and $N_{\sigma}(C>A)$.
It is by definition that $D=(A, B, C)$ is balanced if $N_{D}(A>B)=N_{D}(B>C)=N_{D}(C>A)$, and $D=(A, B, C)$ is nontransitive if $N_{D}(A>B), N_{D}(B>C), N_{D}(C>A)>$ $\left(n^{2} / 2\right)$.

Remark 1. $N_{D}(A>B)$ in Definition 4 represents the number of consequences that a die $A$ beats a die $B$ when we consider all possible outcomes when we roll $A$ and $B$. Note that $N_{D}(A>B)$ is the same as the notation $\sum_{s_{i}=A} q_{\sigma(D)}^{+}\left(s_{i}\right)$ in [14].

Example 1. Let $D$ be the following set of 3-sided dice:

$$
\begin{aligned}
& A=9 \quad 51 \text {, } \\
& B=8 \quad 4 \quad 3 \text {, } \\
& C=7 \quad 6 \quad 2 \text {. }
\end{aligned}
$$

Then, $\sigma(D)=A C B B A C C B A$ and $P(A>B)=P(B>C)$ $=P(C>A)=(5 / 9)$. Therefore, this set of dice is balanced and nontransitive.

Definition 5. The concatenation of two words $\sigma$ and $\tau$ is simply the word $\sigma$ followed by $\tau$, denoted by $\sigma \tau$.

We recall a recursive relation of $N(A>B), N(B>C)$, and $N(C>A)$. The following is in the proof of Lemma 2.4 in [14].

Lemma 1 (see (1) in [14]). Let $\sigma$ and $\tau$ be two words that correspond to two sets of dice, respectively. Let $|\sigma|=3 \mathrm{~m}$ and $|\tau|=3 n$ (i.e., corresponding dice are $m$-sided and $n$-sided, respectively). Then, we have

$$
N_{\sigma \tau}(A>B)=N_{\sigma}(A>B)+N_{\tau}(A>B)+m n .
$$

\section{Fair Sets of Dice}

In the following two lemmas "probabilities" mean $P(A>B)$, $P(B>C)$, and $P(C>A)$ and $x, y$, or $z$ is one of $A, B$, or $C$.

Lemma 2. Assume that $\sigma(D)$ has $x y$ and $y x$, i.e., $\sigma(D)=\cdots x y \cdots y x \cdots$. Then, exchanging the orders of $x y$ and $y x$ at the same time, i.e., $\sigma(\widetilde{D})=\cdots y x \cdots x y \cdots$ does not change the "probabilities."

Lemma 3. Assume that $\sigma(D)$ is a word having three different letters, say $x, y$, and $z$. Then, $x y z \sigma(D)$ and $\sigma(D) x y z$ have the same "probabilities" if and only if $\sigma(D)$ has the same numbers of $x, y$, and $z$.

Definition 6. Two words are called similar, $\sigma\left(D_{1}\right) \sim \sigma\left(D_{2}\right)$, if $\sigma\left(D_{1}\right)$ can be rewritten to $\sigma\left(D_{2}\right)$ by exchanging the orders as allowed by virtue of Lemmas 2 and 3 .

Note that, if $\sigma\left(D_{1}\right) \sim \sigma\left(D_{2}\right)$, then two words have the same length and $P_{D_{1}}(A>B)=P_{D_{2}}(A>B)$, $P_{D_{1}}(B>C)=P_{D_{2}}(B>C)$, and $P_{D_{1}}(C>A)=P_{D_{2}}(C>A)$. 
Example 2. Lemmas 2 and 3 indicate that $\cdots x y \cdots y x \cdots \sim \cdots y x \cdots x y \cdots$ and $x y z \sigma(D) \sim \sigma(D) x y$ $z$ if and only if $|x|_{\sigma(D)}=|y|_{\sigma(D)}=|z|_{\sigma(D)}$.

Conjecture 1. (fair dice). If $D$ is fair, then the length of $D$ is a multiple of 6 and it is similar to an iterated concatenation of $x y z z y x$.

Note that $\tau:=A B C C B A$ is a fair set of 2-sided dice, which will be used in Section 4 .

Example 3. Let $\sigma(D)$ be $A A B B C C C C B B A A$. It is fair and

$A A B B C C C C B B A A \sim A B A B C C C C B A B A \sim A B A C B C C B C$ $A B A \sim A B C A B C C B A C B A \sim A B C C B A A B C C B A=(A B$ $C C B A)^{2}$.

To support Conjecture 1, let us consider only two-die case, i.e., a fair word with only two letters $A$ and $B$. In this case, a set of dice and a corresponding word are called fair if $P(A>B)=P(B>A)=(1 / 2)$.

Theorem 1. Assume that a given fair word $\sigma_{f}$ has $4 \mathrm{~m}$ letters with $|A|=|B|=2 m$. Then, $\sigma_{f} \sim(A B B A)^{m}$.

Proof. We may assume that $\sigma_{f}$ starts with $A$ (if not, we exchange the notations of $A$ and $B$ ). Then, let us claim that the factor $A B B A$ can be extracted to the front without changing probabilities, i.e., $\sigma_{f} \sim A B B A \omega_{f}$, where $\omega_{f}$ is a fair word having $4(m-1)$ letters with $|A|=|B|=2(m-1)$. Mathematical induction with this fact leads us to the result that $\sigma_{f} \sim(A B B A)^{m}$.

Observe first that $\sigma_{f} \sim A B \omega_{0}$, where $\omega_{0}$ is a word such that $A B \omega_{0}$ is fair. If $\sigma_{f}=A B \omega_{0}$, then we are done. Suppose not, i.e., $\sigma_{f}=A \cdots A B \omega_{1}$. We would like to see that $\omega_{1}$ contains a subword $B A$. If $\omega_{1}$ would not have $B A$, then the given fair dice should be expressed by $\sigma_{f}=A \cdots A B A \cdots A B \cdots B$, which is not fair at all. Due to having $B A$ in $\omega_{1}$, exchanging $A B$ and $B A$ together will hold probabilities, i.e., $\sigma_{f} \sim A \cdots B A \omega_{2}$, where $\omega_{2}$ is the word obtained from $\omega_{1}$ by replacing $B A$ by $A B$. A similar argument works to reveal that $\omega_{2}$ has $B A$ and therefore $\sigma_{f} \sim A \cdots B A A \omega_{3}$. Keep doing this procedure until $\sigma_{f} \sim A B \omega_{0}$.

Next see that $\sigma_{f} \sim A B B A \omega_{f}$ as claimed. If $\omega_{0}$ starts with $B A$, then we are done. If not, there are three cases, that is, $\omega_{0}$ is one of $A B \omega_{4}, A \cdots A B \omega_{5}, B \cdots B A \omega_{6}$. Since a similar argument can be applied, let us consider the first case when $\omega_{0}=A B \omega_{4}$. If $\omega_{4}$ would not have $B A$, then it should be expressed by $A \cdots A B \cdots B$. This means that $\sigma_{f} \sim A B A B A \cdots A B \cdots B$, which is impossible to be fair. So, $\omega_{4}$ contains a subword $B A$. Exchanging $B A$ (in $\omega_{4}$ ) for $A B$ (in front of $\omega_{4}$ ) shows that $\sigma_{f} \sim A B B A \omega_{f}$. As mentioned, in the other two cases, a similar argument reveals that $\sigma_{f} \sim A B B A \omega_{f}$ as claimed.

\section{Irreducible Sets of Dice}

In this section, we construct irreducible balanced nontransitive sets of $n$-sided dice for any positive integer $n$. Note that this answers Question 5.2 in [14].

Definition 7. A balanced nontransitive word (and a corresponding set of dice) is called irreducible if there do not exist balanced nontransitive words $\sigma_{1}$ and $\sigma_{2}$ (both nonempty) such that $\sigma=\sigma_{1} \sigma_{2}$.

Lemma 4. Let $\sigma$ be a balanced nontransitive word and $\tau=A B C C B A$. Then, $\tau \sigma$ is balanced and nontransitive.

Proof. $\tau$ is balanced but not nontransitive since $P(A>B)=P(B>C)=P(C>A)=(1 / 2)$. Lemma 2.4 in [14] implies that $\tau \sigma$ is balanced. It remains to prove that it is nontransitive. Let $|\sigma|=3 n$. Equation (2) implies

$$
N_{\tau \sigma}(A>B)=2+N_{\sigma}(A>B)+2 n>2+\frac{n^{2}}{2}+2 n=\frac{(n+2)^{2}}{2} \text {. }
$$

Similarly, we have $N_{\tau \sigma}(B>C), N_{\tau \sigma}(C>A)>\left((n+2)^{2} /\right.$ 2). Therefore, $\tau \sigma$ is also nontransitive (Definition 4$)$.

Lemma 5. Let $\sigma$ be an irreducible balanced nontransitive word that corresponds to a set of either 3-sided or 4-sided dice and $\tau=A B C C B A$. Then, $(\tau)^{k} \sigma$ is an irreducible balanced nontransitive word.

Proof. Applying Lemma $4 k$ times, we conclude that $(\tau)^{k} \sigma$ is balanced and nontransitive. It remains to prove that it is irreducible. Suppose that $(\tau)^{k} \sigma$ is reducible. Then, we can write $(\tau)^{k} \sigma$ as $\pi \pi^{\prime}$ where $\pi$ is an irreducible balanced nontransitive word and $\pi^{\prime}$ is a balanced nontransitive word. Since $(\tau)^{n}$ is fair (so not nontransitive) and $(\tau)^{n} A B C$ is not balanced for any nonnegative integer $n, \pi$ should be of the form $(\tau)^{k} \pi^{\prime \prime}\left(\pi^{\prime \prime}\right.$ is not empty). We write $(\tau)^{k} \sigma$ as $(\tau)^{k} \pi^{\prime \prime} \pi^{\prime \prime \prime}$. Since $\left|\pi^{\prime \prime \prime}\right|<12, \pi^{\prime \prime \prime}$ is irreducible. This implies that $\sigma=\pi^{\prime \prime} \pi^{\prime \prime \prime}$ corresponds to a balanced nontransitive set of 4-sided dice and it contains a balanced nontransitive word $\pi^{\prime \prime \prime}$ of length less than 12 , which contradicts that $\sigma$ is irreducible.

We are now ready to construct an irreducible balanced nontransitive set of $n$-sided dice.

Theorem 2. For any $n \geq 3$, there exists an irreducible balanced nontransitive set of $n$-sided dice.

Proof. We first consider the following two sets of dice: 


$$
\begin{array}{lllll}
A_{3}=9 & 5 & 1, & \\
B_{3}=8 & 4 & 3, & \\
C_{3}=7 & 6 & 2, & \\
A_{4}=10 & 7 & 5 & 4, \\
B_{4}=12 & 9 & 3 & 2, \\
C_{4}=11 & 8 & 6 & 1 .
\end{array}
$$

Both $D_{3}=\left(A_{3}, B_{3}, C_{3}\right)$ and $D_{4}=\left(A_{4}, B_{4}, C_{4}\right)$ are irreducible, balanced, and nontransitive. As before, we consider $\tau=A B C C B A$. We now construct an irreducible balanced nontransitive set of $n$-sided dice for any $n \geq 3$. We already constructed such a set of dice when $n=3$ and $n=4$. When $n \geq 5$ is odd, we write $n=3+2 k, k \geq 1$. Then, $(\tau)^{k} \sigma\left(D_{3}\right)$ is an irreducible balanced nontransitive set of $n$-sided dice due to Lemma 5. When $n \geq 5$ is even, we write $n=4+2 k, k \geq 1$. Then, $(\tau)^{k} \sigma\left(D_{4}\right)$ is an irreducible balanced nontransitive set of $n$-sided dice due to Lemma 5 .

\section{Possible Probability}

Let $(A, B, C)$ be a balanced nontransitive set of $n$-sided dice with $P(A>B)=P(B>C)=P(C>A)>(1 / 2)$. In this section, our interest is in a possible probability $P(A>B)$. Let us state what is on our mind.

Conjecture 2. If $(A, B, C)$ is a balanced nontransitive set of $n$-sided dice such that $P(A>B)>(1 / 2)$, then

$$
\left(\frac{1}{2}<\right) P(A>B)<\frac{1}{2}+\frac{1}{9} .
$$

5.1. Probabilities of Our Construction in Theorem 2. To support Conjecture 2, we first calculate all possible probabilities of the set of dice which are constructed in Section 4.

Lemma 6. Let $\sigma$ (resp. $\tau$ ) be a word of length $3 m$ (resp. 3n) that corresponds to a set of dice. Then,

$$
P_{\sigma \tau}(A>B)=\frac{1}{2}+\frac{\left(N_{\sigma}(A>B)-\left(m^{2} / 2\right)\right)+\left(N_{\tau}(A>B)-\left(n^{2} / 2\right)\right)}{(m+n)^{2}} \text {. }
$$

Similarly, we have

$$
\begin{aligned}
P_{\sigma \tau}(B>C) & =\frac{1}{2}+\frac{\left(N_{\sigma}(B>C)-\left(m^{2} / 2\right)\right)+\left(N_{\tau}(B>C)-\left(n^{2} / 2\right)\right)}{(m+n)^{2}}, \\
P_{\sigma \tau}(C>A) & =\frac{1}{2}+\frac{\left(N_{\sigma}(C>A)-\left(m^{2} / 2\right)\right)+\left(N_{\tau}(C>A)-\left(n^{2} / 2\right)\right)}{(m+n)^{2}} .
\end{aligned}
$$

Proof. It is an easy consequence of equation (2) since $N_{\sigma \tau}(A>B)=(m+n)^{2} P_{\sigma \tau}(A>B)$.
Remark 2. Note that $N_{\sigma}(A>B)$ represents the number of consequences that a die $A$ beats a die $B$. Therefore, $\left(N_{\sigma}(A>B)-\left(m^{2} / 2\right)\right)$ in Lemma 5.2 represents how far $\sigma$ is from being fair. For example, if $N_{\sigma}(A>B)-\left(m^{2} / 2\right)=0$, then $P_{\sigma}(A>B)=(1 / 2)$.

Lemma 7. Let $\sigma$ and $\tau$ be as in Lemma 6. Assume that $(1 / 2)<P_{\sigma}(A>B)<a$ and $(1 / 2)<P_{\tau}(A>B)<b$. Then, $(1 / 2)<P_{\sigma \tau}(A>B)<\max \{a, b\}$.

Proof. Equation (2) with $N_{\sigma \tau}(A>B)=(m+n)^{2} P_{\sigma \tau}(A>B)$, $N_{\sigma}(A>B)=m^{2} P_{\sigma}(A>B)$ and $N_{\tau}(A>B)=n^{2} P_{\tau}(A>B)$ implies

$$
\begin{aligned}
& (m+n)^{2} P_{\sigma \tau}(A>B)=m^{2} P_{\sigma}(A>B)+n^{2} P_{\tau}(A>B)+m n \\
& <\max \{a, b\}\left(m^{2}+n^{2}+\frac{1}{\max \{a, b\}} m n\right)<\max \{a, b\}(m+n)^{2},
\end{aligned}
$$

since $(1 / 2)<\max \{a, b\}$.

We are now ready to calculate the probability $P(A>B)$. Let $\sigma$ be a word that is constructed in Theorem 2. Then, $\sigma$ is either $(\tau)^{k} \tau_{1}$ or $(\tau)^{k} \tau_{2}$ for some nonnegative integers $k$, where

$$
\begin{aligned}
& D\left(\tau_{1}\right)=\left\{\begin{array}{lll}
9 & 5 & 1 \\
8 & 4 & 3 \\
7 & 6 & 2
\end{array}\right. \\
& D\left(\tau_{2}\right)=\left\{\begin{array}{llll}
10 & 7 & 5 & 4 \\
12 & 9 & 3 & 2 \\
11 & 8 & 6 & 1
\end{array}\right. \\
& D(\tau)= \begin{cases}6 & 1 \\
5 & 2 \\
4 & 3\end{cases}
\end{aligned}
$$

Note that $(\tau)^{k}$ is a fair word (i.e., $\left.P_{\tau^{k}}(A>B)=(1 / 2)\right)$. For $i=1,2$, Lemma 2.1 implies that

$$
\begin{aligned}
\left(2 k+n_{i}\right)^{2} P_{\sigma}(A>B) & =(2 k)^{2} P_{\tau^{k}}(A>B)+\left(n_{i}\right)^{2} P_{\tau_{i}}(A>B)+2 k n_{i} \\
& =\frac{(2 k)^{2}}{2}+\left[\frac{n_{i}^{2}+2}{2}\right]+2 k n_{i}=\left[\frac{\left(n_{i}+2 k\right)^{2}+2}{2}\right],
\end{aligned}
$$

which is the closest integer greater than $\left(\left(n_{i}+2 k\right)^{2} / 2\right)$. Here, $3 n_{i}$ is the length of $\tau_{i}$ and, therefore, $n_{i}=i+2$.

Therefore, Conjecture 2 is true in this case.

Remark 3. Similarly, we can also calculate possible probabilities of the set of dice that is constructed in [14]. Let $\sigma$ be a word that is constructed in Theorem 2.1 of [14]. Then, $\sigma$ is a product of several $\tau_{1}, \tau_{2}$, and $\tau_{3}$, where 


$$
\begin{aligned}
& D\left(\tau_{1}\right)=\left\{\begin{array}{lllll}
9 & 5 & 1 \\
8 & 4 & 3, \\
7 & 6 & 2
\end{array}\right. \\
& D\left(\tau_{2}\right)=\left\{\begin{array}{ccccc}
12 & 10 & 3 & 1 \\
9 & 8 & 7 & 2, \\
11 & 6 & 5 & 4
\end{array}\right. \\
& D\left(\tau_{3}\right)=\left\{\begin{array}{lllll}
15 & 11 & 7 & 4 & 3 \\
14 & 10 & 9 & 5 & 2 \\
13 & 12 & 8 & 6 & 1
\end{array}\right.
\end{aligned}
$$

$P_{\tau_{i}}(A>B)=\left(\left[n_{i}^{2}+2 / 2\right] / n_{i}^{2}\right)$ for $i=1,2,3$, where $n_{i}=i+2$. Therefore, Lemma 7 implies that $P_{\sigma}(A>B) \leq$ $(5 / 9)=(1 / 2)+(1 / 18)$. Therefore, Conjecture 2 is true in this case.

5.2. Best Bound for Probability. We first show that $(1 / 2)$ is the greatest lower bound of $P(A>B)$, which means that the inequality $P(A>B)>(1 / 2)$ is optimal.

Theorem 3. There exists a balanced nontransitive set of dice such that $P(A>B)-(1 / 2)>0$ becomes arbitrary small.

Proof. Let $n=2 m+1(m \in \mathbb{N})$. Then, we construct a word $\sigma$ (or a corresponding set of dice) as follows. First, consider the word $(A B C C B A)^{m}(B A C)$ which is not balanced, since the number of the events for $C$ to beat $A$ or $B$ is one more than the ones of the events for $A$ or $B$ to beat $C$ (therefore, $C$ always beats both $A$ and $B$ at least in probability). To change this word to a balanced one, let us replace $B C$ by $C B$ on the first factor $A B C C B A$. In all, the word $\sigma$ obtained by

$$
\sigma:=(A C B C B A)(A B C C B A)^{m-1}(B A C)
$$

is balanced. A direct computation of $P(A>B)$ on $\sigma$, or more precisely,

$$
\begin{aligned}
N_{\sigma(D)}(A>B)= & 0+2+\{(2+4)+\cdots+(2(m-1)+2 m)\} \\
& +(2 m+1)=2 m^{2}+2 m+1
\end{aligned}
$$

shows that

$$
P(A>B)=\frac{2 m^{2}+2 m+1}{(2 m+1)^{2}}=\frac{1}{2}+\frac{0.5}{n^{2}},
$$

which goes to $(1 / 2)$ as $n \longrightarrow \infty$. This indicates that $(1 / 2)$ is the greatest lower bound of $P(A>B)$ and the inequality $P(A>B)>(1 / 2)$ could not get better.

For the upper bound of $P(A>B)$, recall that Conjecture 2 states that

$$
P(A>B)<\frac{1}{2}+\frac{1}{9}
$$

To support this inequality, we provide three new constructions of sets of dice of which probability is close to $(1 / 2)+(1 / 9)$. For simplicity, assume that $n=6 p$ (later, we discuss when $n=6 p+2$ or $n=6 p+4$ which is similar to the present case). Let us start with the most unmixed fair $n$-sided dice $\sigma_{f}$,

$$
\sigma_{f}:=A \cdots A B \cdots B C \cdots C C \cdots C B \cdots B A \cdots A,
$$

where "..." means that all the letters are the same as the boundary letters and the numbers of letters in "..." are the same, i.e., $|A \cdots A|=|B \cdots B|=|C \cdots C|=3 p$.

We apply the following algorithm to construct the largest probability $P(A>B)$.

\section{Algorithm 1}

Step 1: if there is no triple $A B, B C, C A$ in a word, then replace $x y$ and $y x$ in a word for $x, y \in\{A, B, C\}$ so that the resulting word contains $A B, B C, C A$.

Step 2: if you find $A B, B C, C A$, then replace $A B, B C, C A$ by $B A, C B, A C$, respectively.

\section{Remark 4}

Replacement in Step 1 does not change the probability due to Lemma 2.

Replacement in Step 2 increases the number of events for $A$ (resp. $B$ or $C$ ) to beat $B$ (resp. $C$ or $A$ ) by 1 . In particular, doing this replacement keeps the words balanced and nontransitive. Therefore we may expect that keeping replacing in this way would lead the largest probability of $P(A>B)$.

Since there is no $C A$ in $\sigma_{f}$, we first apply Step 1 (exchange of $B C$ and $C B$ ) several times to $\sigma_{f}$ in order to obtain a word $\sigma_{1}$ which is still fair but having sequels $C A$ by

$$
\begin{aligned}
\sigma_{1}:= & A \cdots A A \cdots A A \cdots A B \cdots B B \cdots B C \cdots C \\
& C \cdots C C \cdots C \mathbf{B} \cdots \mathbf{B C} \cdots C C \cdots C B \cdots B \\
& B \cdots B B \cdots B \mathbf{C} \cdots \mathbf{C A} \cdots A A \cdots A A \cdots A,
\end{aligned}
$$

(i.e., the last one-third of the first $B \cdots B$ in (17) moves to the middle and the last one-third of the second $C \cdots C$ in (17) does to the front of the second $A \cdots A$ (the moved ones are in bold). Here, $|A \cdots A|=|B \cdots B|=|C \cdots C|=p$ in (18). Therefore, the numbers of replacing $B C$ and $C B$ by $C B$ and $B C$, respectively, are the same as $3 p^{2}$.)

We now apply Step $23 p^{2}$ times to obtain a balanced nontransitive word $\sigma_{2}$ by

$$
\begin{aligned}
\sigma_{2}:= & \mathbf{B} \cdots \mathbf{B} A \cdots A A \cdots A A \cdots A C \cdots C C \cdots C \\
& C \cdots C \mathbf{B} \cdots \mathbf{B} B \cdots B C \cdots C C \cdots C B \cdots B \\
& B \cdots B B \cdots B A \cdots A A \cdots A A \cdots A \mathbf{C} \cdots \mathbf{C},
\end{aligned}
$$

(i.e., in (18), the first $B \cdots B$ moves in front, the second $B \cdots B$ does to almost middle, and the last $C \cdots C$ does to the end. The moved ones are in bold as before). Here, the probability $P(A>B)$ of $\sigma_{2}$ increases to $(1 / 2)+\left(3 p^{2} /(6 p)^{2}\right)=(7 / 12)$.

Since there is no $A B$ and $C A$ in $\sigma_{2}$, we apply Step $16 p$ times as follows; we move $B$ and $C$ in $\sigma_{2}$ to the ones in $\sigma_{3}$ (all 
of which are in bold) in order to produce the products $A B$ and $C A$.

$$
\begin{aligned}
\sigma_{2}:= & B \cdots B A \cdots A A \cdots A A \cdots A C \cdots C C \cdots C \\
& C \cdots C \mathbf{B} \cdots B B \cdots B C \cdots C C \cdots \mathbf{C} B \cdots B \\
& B \cdots B B \cdots B A \cdots A A \cdots A A \cdots A C \cdots C, \\
\sigma_{3}:= & B \cdots B A \cdots A A \cdots A A \cdots A \mathbf{B} C \cdots C C \cdots C \\
& C \cdots C \dot{B} \cdots \ddot{B} \ddot{B} \cdots \ddot{B} \ddot{C} \cdots \ddot{C} \ddot{C} \cdots \dot{C} B \cdots B \\
& B \cdots B B \cdots B C A \cdots A A \cdots A A \cdots A C \cdots C \\
& (\dot{B} \text { and } \dot{C} \text { have been moved to } \mathbf{B} \text { and } \mathbf{C}, \text { respectively). }
\end{aligned}
$$

This means that we need to exchange $B C$ to $C B$ in the middle part (which is blue and has two dots above the character on $\sigma_{3}$ ) $6 p$ times ( $3 p$ to move $B$ and another $3 p$ to beat $C)$. Then, to increase $P(A>B)$, one more exchange from $B C$ to $C B$ in the middle part is necessary.

In addition to this observation, we would like to measure how many times we can apply Step 2 to $\sigma_{3}$ in terms of $p$ (this is because the denominator of $P(A>B)$ is $(6 p)^{2}$, so we would better express in $p$ in order to examine asymptotic behavior of $P(A>B))$. Put by $m$ the number of further replacement such that $m$-many $B$ 's move between $B \cdots B$ and $A \cdots A$ in front. For this, we need extra $3 p m$-many exchanges of $B C$ and $C B$ in the middle part. Since the remaining of each $B$ 's or $C$ 's in the middle is $2 p-m$, the possible exchange of $B C$ to $C B$ is $(2 p-m)^{2}$, which should be at least $9 p m(=3 p m+3 p m+3 p m)$ (the last $3 p m$ is due to move $m$-many $B$ 's in the further replacement). As a summary, we have that

$$
\begin{gathered}
(2 p-m)(2 p-m) \geq 9 p m(m \leq 2 p) \\
\Longrightarrow m \leq \frac{13-\sqrt{154}}{2} p
\end{gathered}
$$

Therefore, the largest probability on $P(A>B)$ in the argument above is

$$
\frac{1}{2}+\frac{3 p^{2}+(13-\sqrt{154} / 2) p \cdot 3 p}{(6 p)^{2}}=\frac{1}{2}+\frac{15-\sqrt{154}}{24} \approx \frac{1}{2}+\frac{1}{9.25}<\frac{1}{2}+\frac{1}{9} .
$$

Note that the maximum $m$ (in $\mathbb{N}$ ) means that, since all $B C$ are exhausted in the last word, the probability $P(A>B)$ is not able to be larger in this construction.

The cases when $n=6 p+2$ and $n=6 p+4$ can be investigated by a similar procedure. By applying Step 1 (or exchanging $B C$ by $C B$ ) and Step 2 (or replacing $A B, B C$, and $C B$ by $B A, C B$, and $B C)$, the probability $P(A>B)$ increases by

$$
\begin{gathered}
\frac{p(3 p+1)}{[2(3 p+1)]^{2}}, \quad(\text { when } n=6 p+2) \\
\text { or } \frac{p(3 p+2)}{[2(3 p+2)]^{2}}, \quad(\text { when } n=6 p+4),
\end{gathered}
$$

(which is similar to the increase $p(3 p) /(6 p)^{2}$ when $n=6 p$ ). In these cases, after doing this procedure, new words are expressed by

$$
\begin{aligned}
n= & 6 p+2: \sigma_{2}=B \cdots B A \cdots A A \cdots A A \cdots A A B C \cdots C C \cdots C \\
& C \cdots C C B \cdots B B \cdots B C \cdots C C \cdots C C \cdots B \\
& B \cdots B B \cdots B B A \cdots A A \cdots A A \cdots A A C \cdots C, \\
n= & 6 p+4: \sigma_{2}^{\prime}=B \cdots B A \cdots A A \cdots A A \cdots A A A B B C \cdots C C \cdots C \\
& C \cdots C C C B \cdots B B \cdots B C \cdots C C \cdots C C C \cdots B \\
& B \cdots B B \cdots B B B A \cdots A A \cdots A A \cdots A A A C \cdots C .
\end{aligned}
$$

Here, each $\cdots$ has exactly $p-2$ letters. For example, $|A \cdots A|=p-2+2=p$.

Next, to make the largest $P(A>B)$, we do Step 2 further as in the previous case. Shortly speaking, when $n=6 p+2$, we have that

$$
\begin{gathered}
(2 p-m)(2 p+1-m)-(3 p+1) \geq 3 m(3 p+1)(m \leq 2 p) \\
\Leftrightarrow m^{2}-(13 p+4) m+\left(4 p^{2}-p-1\right) \geq 0 \\
\Rightarrow m \leq \frac{13 p+4-\sqrt{153 p^{2}+108 p+20}}{2}
\end{gathered}
$$

Hence, the increased probability on $P(A>B)$ in the argument (i.e., except 1/2) is

$$
\frac{\left(p+\left(\left(13 p+4-\sqrt{153 p^{2}+108 p+20}\right) / 2\right)\right)(3 p+1)}{(6 p+2)^{2}} \stackrel{p}{\longrightarrow} \rightarrow \infty \frac{1}{12}+\frac{13-\sqrt{153}}{24}<\frac{1}{9.12}<\frac{1}{9} .
$$


Note that, since the numerator $13 p+4-\sqrt{153 p^{2}+108 p+20}$ is increasing in $p$, the last inequality holds for all $p$.

Similarly, when $n=6 p+4$, we have that

$$
\begin{gathered}
(2 p-m)(2 p+2-m)-2(3 p+2) \geq 3 m(3 p+2)(m \leq 2 p) \\
\Leftrightarrow m^{2}-(13 p+8) m+\left(4 p^{2}-2 p-4\right) \geq 0 \\
\Rightarrow m \leq \frac{13 p+8-\sqrt{153 p^{2}+216 p+80}}{2} .
\end{gathered}
$$

Hence, the increased probability on $P(A>B)$ for $n=$ $6 p+4$ (again except $1 / 2$ ) is

$$
\frac{\left(p+\left(13 p+8-\sqrt{153 p^{2}+216 p+80} / 2\right)\right)(3 p+2)}{(6 p+4)^{2}} \stackrel{p}{\longrightarrow} \rightarrow \infty \frac{1}{12}+\frac{13-\sqrt{153}}{24}<\frac{1}{9.12}<\frac{1}{9}
$$

Note again that the numerator $13 p+8-\sqrt{153 p^{2}+216 p+80}$ increases in $p$.

Let us summarize the argument above. Start with the most unmixed fair word $\sigma_{f}$ (which is (17)). By replacing $A B$, $B C, C A$ by $B A, C B, A C$, i.e., (Step 2 ) as much as possible, the probability $P(A>B)(=P(B>C)=P(C>A))$ increases most. Then, the computation above tells us that

$$
P(A>B)<\frac{1}{2}+\frac{1}{9}
$$

which upholds Conjecture 2.

\section{Remark 5}

(i) Let us tell why we think the last constructed word in this argument provides the largest probability on $P(A>B)$. First, observe that we have considered the only case when $n$ was even (when $n=6 p, 6 p+2$, or $6 p+4)$. We, however, believe that this would be enough due to the following observation. A direct computation shows that there is only one possible probability $P(A>B)$ when $n=3$, or equivalently, any three balanced, nontransitive dice with 3 sides can be expressed by a balanced, nontransitive word which is similar to $\sigma_{3}=C B A B A C A C B$. In this case, $P_{\sigma_{3}}(A>B)=(5 / 9)$. Then, add $C B A$ at the end of $\sigma_{3}$ and then exchange $C A$ (in $\sigma_{3}$ ) to $A C$. Then, the constructed word $\sigma_{4}$ is $\sigma_{4}=C B A B A A C C B C B A$, which becomes balanced and nontransitive. For this, $P_{\sigma_{4}}(A>B)=(9 / 16)$, which is greater than $P_{\sigma_{3}}(A>B)=(5 / 9)$. A similar computation can be done for more general $n$. Therefore, with this trick (however, we do not know if we can do this trick at all times), the probability of $P(A>B)$ would get greater for even $n$ 's than odd ones.

(ii) Second, proving that the abovementioned probability is the largest probability related to construction of all balanced nontransitive sets of dice and it also seems to be related to property of fair sets of dice, i.e., Conjecture 1, and we leave this for future work.

Remark 6. In [13], Schaefer further generalized the results in [14] to sets of $n$-sided $m$ dice for any positive integer $m$. It will be very interesting to generalize our results to sets of $m$ dice for $m \geq 4$ and we also leave this for future work.

\section{Data Availability}

No data were used to support this study.

\section{Conflicts of Interest}

The authors declare that they have no conflicts of interest.

\section{Acknowledgments}

The first author was supported by Basic Science Research Program through the National Research Foundation of Korea (NRF) funded by the Korea government (NRF2019R1F1A1061300). The second author was partially supported by Chonnam National University (Grant no. 2018-0978) and by the National Research Foundation of Korea (NRF) grant funded by the Korea government (MSIP) (no. 2017R1C1B2010081). The authors would like to thank the referees for a number of corrections and very useful suggestions.

\section{References}

[1] M. Gardner, "Mathematical games," Scientific American, vol. 223 , no. 6, pp. 110-115, 1970.

[2] M. Gardner, "Mathematical games," Scientific American, vol. 230, no. 5, pp. 120-125, 1974.

[3] R. P. Savage, "The paradox of nontransitive dice," The American Mathematical Monthly, vol. 101, no. 5, pp. 429-436, 1994. 
[4] L. Angel and M. Davis, "A direct construction of nontransitive dice sets," Journal of Combinatorial Designs, vol. 25, no. 11, pp. 523-529, 2017.

[5] J. Buhler, A. Gamst, R. Graham, and A. Hales, "Explicit error bounds for lattice edgeworth expansions," in Connections in Discrete Mathematics: A Celebration of the Work of Ron Graham, S. Butler, J. Cooper, and G. Hurlbert, Eds., pp. 321-352, Cambridge University Press, Cambridge, UK, 2018.

[6] J. Buhler, R. Graham, and A. Hales, "Maximally nontransitive dice," The American Mathematical Monthly, vol. 125, no. 5, pp. 387-399, 2018.

[7] B. Conrey, J. Gabbard, K. Grant, A. Liu, and K. E. Morrison, "Intransitive dice," Mathematics Magazine, vol. 89, no. 2, pp. 133-143, 2016.

[8] C. Cooley, W. Ella, M. Follett, E. Gilson, and L. Traldi, "Tied dice. II. some asymptotic results," Journal of Combinatorial Mathematics and Combinatorial Computing, vol. 90, pp. 241-248, 2014.

[9] E. Gilson, C. Cooley, W. Ella, M. Follett, and L. Traldi, “The efron dice voting system," Social Choice and Welfare, vol. 39, no. 4, pp. 931-959, 2012.

[10] J. Grime, "The bizarre world of nontransitive dice: games for two or more players," The College Mathematics Journal, vol. 48, no. 1, pp. 2-9, 2017.

[11] B. G. Kronenthal and L. Traldi, "Tied dice," Journal of Combinatorial Mathematics and Combinatorial Computing, vol. 68, pp. 85-96, 2009.

[12] C. M. Rump, "Strategies for rolling the efron dice," Mathematics Magazine, vol. 74, no. 3, pp. 212-216, 2001.

[13] A. Schaefer, "Balanced non-transitive dice II: tournaments," 2017, https://arxiv.org/abs/1706.08986.

[14] A. Schaefer and J. Schweig, "Balanced nontransitive dice," The College Mathematics Journal, vol. 48, no. 1, pp. 10-16, 2017. 